\title{
An Epidemiologic Study of the Association between Free Recall Dichotic Digits Test Performance and Vascular Health
}

DOI: $10.3766 /$ jaaa.17079

\author{
Mary E. Fischer* \\ Karen J. Cruickshanks* ${ }^{*}$ \\ Lauren K. Dillard \\ David M. Nondahl* \\ Barbara E. K. Klein* \\ Ronald Klein* \\ James S. Pankow§ \\ Ted S. Tweed* \\ Carla R. Schubert* \\ Dayna S. Dalton* \\ Adam J. Paulsen*
}

\begin{abstract}
Background: Associations between vascular health-related factors and hearing loss defined using audiometric pure-tone thresholds have been found. Studies have not focused on a potential relationship between vascular health-related factors and central auditory processing.
\end{abstract} Purpose: The aim of this study was to evaluate, on a population level, the relationship of vascular health-
related factors with central auditory function.

Research Design: A cross-sectional, population study.

Study Sample: Subjects were participants in the Epidemiology of Hearing Loss Study (EHLS) or the Beaver Dam Offspring Study (BOSS) — prospective studies of aging and sensory loss. BOSS participants were the adult offspring of participants in the EHLS. Participants who completed the Dichotic Digits Test (DDT) during the fourth examination period of the EHLS (2008-2010) or the second examination period of the BOSS (2010-2013) were included $(n=3,655$, mean age $=61.1$ years).

Data Collection and Analysis: The DDT-free recall test was conducted using 25 sets of triple-digit pairs
at a $70 \mathrm{~dB} \mathrm{HL}$ presentation level. The total number of correctly repeated digits from the right and left ears
was converted to a percentage correct and used as an outcome. The percentage correct in the left ear
was subtracted from the percentage correct in the right ear and used as an outcome. Vascular health-
related measures obtained during the examination included blood pressure, mean carotid intima-media
thickness, femoral pulse wave velocity (PWV), hemoglobin A1C, and non-high-density lipoprotein (HDL)
cholesterol, and, in the EHLS participants, C-reactive protein and interleukin-6. Information on vascular
health-related history and behaviors was self-reported. General linear modeling produced estimates of
the age- and sex-adjusted least squares means for each vascular factor, and multiple linear regression
was used for multivariable modeling of each outcome.

Results: After multivariable adjustment, participants with diabetes had a significantly lower (worse) mean DDT-free recall total score $(-2.08$ percentage points, $p<0.001)$ than those without diabetes.

*Department of Ophthalmology and Visual Sciences, University of Wisconsin, Madison, WI; †Department of Population Health Sciences, University of Wisconsin, Madison, Wl; $\$$ Department of Communication Sciences and Disorders, University of Wisconsin, Madison, WI; §Division of Epidemiology and Community Health, University of Minnesota, Minneapolis, MN

Corresponding author: Mary E. Fischer, Department of Ophthalmology and Visual Sciences, School of Medicine and Public Health, University of Wisconsin-Madison, Madison, WI 53726-2336; Email: fischer@episense.wisc.edu

The project described was supported by Award Numbers R37AG011099 and R01AG021917 from the National Institute on Aging (to K. J. Cruickshanks), Award Number EY06594 from the National Eye Institute (to R. Klein and B. E. K. Klein), and by unrestricted funds from Research to Prevent Blindness (RPB). The content is solely the responsibility of the authors and does not necessarily reflect the official views of the National Institutes of Health. 


\begin{abstract}
Participants who exercised at least once per week had a significantly higher (better) mean DDT-free recall total score $(+1.07$ percentage points, $p<0.01)$ than those who did not exercise at least once per week. Alcohol consumption was associated with a higher DDT-free recall total score $(+0.15$ percentage points per $+25 \mathrm{~g}$ ethanol, $p<0.01$ ). In multivariable modeling of the right-left ear difference in DDT-free recall scores, participants with a history of cardiovascular disease (CVD) or higher PWV demonstrated significantly larger differences (CVD: +3.11 percentage points, $p=0.02$; PWV: +0.36 percentage points per $1 \mathrm{~m} / \mathrm{sec}$, $p<0.01)$. Higher levels of non-HDL cholesterol were associated with smaller right-left ear differences $(-0.22$ percentage points per $10 \mathrm{mg} / \mathrm{dL}, p=0.01$ ). Adjustment for handedness did not affect the results.
\end{abstract}

Conclusions: Vascular health-related factors may play a role in central auditory function.

Key Words: central auditory function, dichotic listening tests, epidemiology, vascular health-related factors

Abbreviations: $\mathrm{BMI}=$ body mass index; BOSS = Beaver Dam Offspring Study; CRP = C-reactive protein; CVD = cardiovascular disease; DDT = Dichotic Digits Test; EHLS = Epidemiology of Hearing Loss Study; $\mathrm{GHb}=$ glycosylated hemoglobin; HDL = high-density lipoprotein; IL-6 = interleukin-6; IMT = intima-media thickness; PTA = pure-tone average; PWV = pulse wave velocity; REA = right ear advantage

\section{INTRODUCTION}

$\mathrm{C}$ entral auditory processing dysfunction has been found to be related to cognitive dysfunction, including decline, impairment, and dementia (Hällgren et al, 2001; Gates et al, 2002; 2008; 2010; 2011; Idrizbegovic et al, 2013; Fischer et al, 2017). The Dichotic Digits Test (DDT) is one of many measures used to evaluate central auditory processing (Musiek et al, 1991; Gates et al, $2008 ; 2010 ; 2011$ ). In the DDT, single-syllable numbers, typically in double-digit or triple-digit pairs, are presented in both ears concurrently, and in the free recall version of the test, individuals are asked to repeat all numbers. One reason that the DDT, as well as other dichotic speech recognition tests, is particularly cognitively challenging may be because the competing signals result in the suppression of the ipsilateral processing pathways and consequently in more reliance on the contralateral pathways (Hugdahl, 1995).

Older age is strongly related to poor performance on central auditory processing tests (Jerger et al, 1994; Wilson and Jaffe, 1996; Strouse et al, 2000; Hällgren et al, 2001; Gates et al, 2002; 2011; Roup et al, 2006; Fischer et al, 2017) and to a greater right ear advantage (REA) in recall of the presented material (Wilson and Jaffe, 1996; Strouse and Wilson, 1999a; Roup et al, 2006; Hommet et al, 2010; Fischer et al, 2017). A recent population study reported that females and those with a college degree performed significantly better on the DDT-free recall (Fischer et al, 2017). The Blue Mountains Hearing Study also found better performance by females on dichotic listening tests (Golding et al, 2006), but few additional factors have been studied or reported to be related to dichotic testing on a population level.

With respect to the relationship between vascular health-related factors and central auditory processing, a cross-sectional study in the Framingham cohort reported that a history of heart attack or stroke in women and current smoking in men were related to performance on the Synthetic Sentence Identification test, a test with competing message (Gates et al, 1993), and in a recent case-control study of patients with stroke, cases had significantly higher rates of central auditory processing deficits than controls (Koohi et al, 2017). It has also been reported that individuals with type 2 diabetes had higher speech thresholds in the Hearing in Noise Test (Frisina et al, 2006). Associations between vascular healthrelated factors and pure-tone threshold hearing loss have been found as well (Gates et al, 1993; Cruickshanks, Klein, et al, 1998; Bainbridge et al, 2008; Kiely et al, 2012; Cruickshanks et al, 2015; Fischer et al, 2015). Factors found to be associated with the risk of hearing impairment in previous prospective studies include current smoking, waist circumference, and glycosylated hemoglobin (GHb) (Cruickshanks et al, 2015), and subclinical atherosclerosis, as measured by carotid intima-media thickness (IMT) and plaque, and body mass index (BMI) (Fischer et al, 2015). Baseline hypertension has been reported to be related to hearing decline over an 11-year follow-up (Kiely et al, 2012), and findings from cross-sectional investigations have suggested associations between hearing loss and smoking (Cruickshanks, Klein, et al, 1998), coronary heart disease (Gates et al, 1993), and diabetes (Bainbridge et al, 2008). Physical exercise has been found to be inversely related to selfreported hearing problems (Curhan et al, 2013).

The aim of this study was to evaluate, on a population level, the relationship of vascular health-related factors with central auditory function. The total score on the DDT-free recall test was used as the measure of central auditory processing, and vascular health-related history, measurements, and behavioral factors were considered as potential-related factors. The relationship between these same factors and the right-left ear difference on the DDT-free recall test was also evaluated. The results may be useful in planning future prospective investigations of the predictors of central auditory decline or dysfunction. 


\section{METHODS}

\section{Study Population}

Participants were part of the Epidemiology of Hearing Loss Study (EHLS) or the Beaver Dam Offspring Study (BOSS)—prospective longitudinal studies of aging and sensory disorders. Participants from the baseline Beaver Dam Eye Study (1987-1988) were eligible to participate in the EHLS, and there were 3,753 participants with an age range of 48-92 years in the EHLS baseline examination (1993-1995). Follow-up examinations occurred at 5 -year intervals, with $>80 \%$ response rates. The data in the present study are from the fourth examination period of the EHLS (2008-2010). Audiometric testing was administered to 1,549 participants, of which 1,391 completed free recall and right eardirected dichotic digits testing (Fischer et al, 2017). Reasons for not completing dichotic digits testing include hearing loss severity or asymmetry $(\mathrm{n}=103)$, not successfully completing the dichotic digits training exercise ( $n=13)$, requesting to stop $(n=14)$, or other/ unknown reasons $(\mathrm{n}=28)$. Details of the EHLS are found in previous reports (Klein et al, 1991; Cruickshanks, Wiley, et al, 1998; Cruickshanks et al, 2003; 2010).

The adult offspring of the EHLS participants were eligible for the BOSS. At the time of the BOSS baseline examination (2005-2008), there were 3,296 participants with an age range of 21-84 years. During the 5-year follow-up examination (2010-2013), audiometric data were obtained from 2,346 participants and dichotic digits testing data from 2,264 participants. The reasons for not completing dichotic digits testing were ineligibility due to hearing loss severity or asymmetry $(n=28)$, not successfully completing the training $(\mathrm{n}=11)$, requesting to stop $(n=26)$, or other/unknown reasons $(n=17)$. Further details of the BOSS are found in previous reports (Zhan et al, 2010; Nash et al, 2011; Fischer et al, 2013; 2015).

There was a total of 3,655 subjects (1,600 men and 2,055 women) included in the study, with an average age of 61.1 years. Close to $4 \%$ of the study population were aged younger than 40 years, $42 \%$ were $40-59$ years, $45 \%$ were 60-79 years, and $9 \%$ were 80 years of age or older.

Approval for this research was obtained from the Health Sciences Institutional Review Board of the University of Wisconsin, and informed consent was obtained from all participants before each examination.

\section{Measurements}

Participants underwent a series of standardized examinations and interviews, which were conducted by examiners who were trained and certified in all study protocols. Similar methods were used in the EHLS and BOSS.

\section{Audiometric Testing}

Audiometric testing was performed, and hearingrelated medical history questionnaires were administered. Testing was performed in accordance with American Speech-Language-Hearing Association guidelines in compliance with the American National Standards Institute standards (ASHA, 1978; ANSI, 1999; 2010). Otoscopy and tympanometry were performed before pure-tone audiometry. Testing was performed in soundtreated booths using clinical audiometers (calibrated every 6 months), TDH-50P earphones and ER3-A inserts in cases of probable ear canal collapse. There were 49 participants tested using insert earphones in other locations. Pure-tone air-conduction thresholds were obtained in both ears at $0.5,1,2,3,4,6$, and $8 \mathrm{kHz}$, and bone-conduction thresholds were obtained at $0.5,2$, and $4 \mathrm{kHz}$. Masking was used as necessary (Cruickshanks, Wiley, et al, 1998; Cruickshanks et al, 2003). A four-frequency pure-tone average (PTA) at $0.5,1,2$, and $4 \mathrm{kHz}$ was calculated for each ear and used to categorize hearing loss severity of either ear (worse ear) as follows: no loss $=\mathrm{PTA} \leq 25 \mathrm{~dB} \mathrm{HL}(\mathrm{n}=2,412$, mean PTA $=12.8 \mathrm{~dB} \mathrm{HL}$ ); mild loss $=25<\mathrm{PTA} \leq 40 \mathrm{~dB}$ $\mathrm{HL}(\mathrm{n}=799$, mean PTA $=32.1 \mathrm{~dB} \mathrm{HL})$; and moderate or marked loss $=$ PTA $>40 \mathrm{~dB}$ HL $(\mathrm{n}=444$, mean PTA $=$ $49.3 \mathrm{~dB}$ HL). There were 3,067 participants with symmetrical hearing loss severity (both ears were in the same severity group), 221 participants in whom the right ear loss was more severe than the left ear, and 367 participants in whom the left ear loss was more severe than the right ear.

\section{DDT}

To complete the DDT, participants had to accurately complete a training/practice task, in which three examples of single-digit, double-digit, and triple-digit pairs were presented. If the participant was unable to repeat any of the numbers in the single-digit or double-digit pairs or did not attempt to repeat any of the numbers in the triple-digit pairs, the participant was not tested on the DDT. The free recall and right ear-directed recall conditions each consisted of 25 sets of triple-digit pairs (three digits presented to each ear simultaneously) using single-syllable numbers 1 through 10 , excluding 7 , presented at $70 \mathrm{~dB}$ HL. The CD for the test was provided by Dr. Richard Wilson. In the free recall task, which was administered first, the participant was asked to repeat as many of the six digits as possible. The numbers of correctly repeated digits from the right and left ears was added together, and the sum was used as the score for the DDT-free recall. The possible range of the score was 0-150 (75 per ear). The number correct was converted into percentage correct for analysis purposes. The right-left ear difference was calculated by 
subtracting the left ear percentage correct from the right ear percentage correct.

\section{Vascular Health-Related Factors}

A history of cardiovascular disease (CVD) was defined as a self-report of a physician-diagnosed stroke, angina, or myocardial infarction. Hypertension was defined as having received a diagnosis of hypertension with current medication use or having a measured systolic blood pressure $\geq 140 \mathrm{mmHg}$ or diastolic blood pressure $\geq 90 \mathrm{mmHg}$. Diabetes was defined as a self-report of a physician diagnosis or a suspected diagnosis with current treatment, or as having a hemoglobin A1C $\geq 6.5 \%$. The assay used to determine hemoglobin A1C used the Tosoh HPLC G7 Glycohemoglobin Analyzer (Tosoh Medics, Inc., San Francisco, CA) with nonfasting venous blood specimens. Cholesterol and high-density lipoprotein (HDL) cholesterol assays were performed using the Roche Modular P Chemistry Analyzer (Roche Diagnostics Corporation, Indianapolis, IN), and nonHDL cholesterol was determined by subtracting the HDL value from the total cholesterol value. BMI was calculated from measures of height and weight $\left(\mathrm{kg} / \mathrm{m}^{2}\right)$, and obesity was defined as $\geq 30 \mathrm{~kg} / \mathrm{m}^{2}$.

The mean carotid IMT and the plaque score were determined through high-resolution B-mode carotid artery ultrasound (MyLab25; Esaote North America Inc., Indianapolis, IN). The IMT was measured in 1.0 $\mathrm{cm}$ segments of the near and far walls of the common artery, internal artery, and the carotid bifurcation. Ultrasound imaging and grading used a modified Atherosclerosis Risk in Communities protocol (Bond et al, 1991; Riley et al, 1991), and the mean of the 12 walls was calculated for the IMT measure. The mean IMT was categorized according to tertiles of the distribution as (1) $\leq 0.6224 \mathrm{~mm},(2)>0.6224 \mathrm{~mm}$ and $\leq 0.7783 \mathrm{~mm}$, and (3) $>0.7783 \mathrm{~mm}$. Plaque was considered to be present if acoustic shadowing was seen in association with at least one of the following, or if acoustic shadowing was not seen with at least two of the following: change in wall shape (protrusion into lumen), change in wall texture, or IMT $>1.5 \mathrm{~mm}$. The plaque score was defined as the number of sites (0-6) with plaque present (Zhong et al, 2012; Schubert et al, 2015). Femoral pulse wave velocity (PWV) was measured using the Complior SP System (Artech Medical/Alam Medical, Vincennes, France). The PWV from the right common carotid artery to the right femoral artery was measured at a time when the signal was clean, had a fast rising systole, sufficient systolic amplitude, a relatively flat diastole for at least ten cycles, and, when possible, a PWV tolerance $<5 \%$ (Zhong et al, 2014; Schubert et al, 2015).

In EHLS participants, the C-reactive protein (CRP) level was determined (Advanced Research and Diagnostic Laboratory, University of Minnesota, Minneapolis,
MN) using a latex particle-enhanced immunoturbidimetric assay from Roche Diagnostics on the Roche Modular P Chemistry Analyzer (Roche Diagnostics Corporation) and was categorized into the following groups: $<1.0,1.0-3.0$, and $>3.0 \mathrm{mg} / \mathrm{L}$. Serum interleukin-6 (IL-6) was measured using the quantitative sandwich enzyme technique of the enzyme-linked immunosorbent assay (QuantiKine High Sensitivity kit; R\&D Systems, Minneapolis, MN) and was categorized into the following tertiles based on the distribution: $\leq 1.192,>1.192$ and $\leq 2.252$, and $>2.252 \mathrm{pg} / \mathrm{mL}$. A summary measure of the number of inflammatory markers in the highest category was created (range: 0-2). Additional details of the CRP and IL-6 assays have been reported (Nash et al, 2013; Wichmann et al, 2014).

Self-reported smoking status was classified as nonsmoker ( $<100$ cigarettes in lifetime), ex-smoker, or current smoker. Alcohol consumption was measured by a quantity/frequency questionnaire and converted to grams of ethanol per week. Ever having consumed heavy alcohol was defined as ever drinking $4+$ drinks per day. Exercise was defined as being sufficient to work up a sweat at least once a week.

\section{Covariates}

Demographic factors used in the analysis were age, sex, and education. Education was categorized as less than a high school graduate ( $<12$ years), a high school graduate (12 years), some college (13-15 years), and college graduate or beyond (16+ years).

Handedness was determined by asking "Are you right or left handed?" with possible answers of "Left," "Right," "Use both equally," or "Unknown." Cognition was measured with the Mini-Mental State Examination test (Folstein et al, 1975). The test was administered to participants aged 50 years and older; participants younger than 50 years were assumed to have no cognitive impairment. If the Mini-Mental State Examination score was $<24$ (of a maximum of 30), or if there was a self- or proxy-reported history of dementia or Alzheimer's disease, the subject was considered to have cognitive impairment.

\section{Statistical Analyses}

All analyses were completed using SAS version 9.4 software (SAS Institute, Inc., Cary, NC).

General linear modeling was used to assess the ageand sex-adjusted associations of the categorically measured vascular health-related factors with the DDT-free recall total score and to estimate age- and sex-adjusted least squares means. Categorical covariates were modeled using indicator variables. To allow for estimates proportional to the observed margins, the ObsMargins option was used. Multiple linear regression was performed to 
evaluate the age- and sex-adjusted associations of the continuously measured factors with the DDT-free recall total score.

Multiple linear regression was used for multivariable modeling, with the significant factors from the age- and sex-adjusted models included in the full multivariable model. The covariates previously identified as being significantly related to the DDT-free recall total score, namely age, sex, education, hearing loss severity, and cognitive impairment (Fischer et al, 2017), were also included in the multivariable model. Indicator variables were used in the models for the education groups, with the college graduate category as the reference group. Indicator variables were also used for the hearing loss severity groups, with the no loss group as the reference. Cognitive impairment was a dichotomous variable. A final, reduced model was developed through the elimination of nonsignificant independent variables.

The modeling approach used for the DDT-free recall total score was followed for the right-left ear difference in percentage correct as the outcome.

\section{RESULTS}

$\mathrm{T}$ he range of the free recall scores was $21.3 \%$ to $100 \%$, with a mean of $76.7 \%$. Additional descriptive information on the study population and the distribution of the DDT scores has been reported (Fischer et al, 2017). In age- and sex-adjusted models, participants with a reported history of CVD had a mean total free recall score that was 2.0 percentage points lower $(p<0.01)$ than participants without CVD, and those with diabetes had an average total free recall score that was 3.0 percentage points lower $(p<0.0001)$ than those without diabetes (Table 1). Hypertension was not strongly related to the total score. Obesity was significantly $(p<0.001)$ associated with the total DDT-free recall score whereby participants who were obese had a mean score of 1.3 percentage points lower than the nonobese. Physical exercise was found to be related to a higher adjusted mean score $(77.6 \%$ in those who exercised at least once/week versus $75.5 \%$ in those who did not; $p<0.0001$ ), but smoking was not related. A history of heavy alcohol use was negatively associated $(-1.9$ percentage points) with DDT-free recall performance, whereas current alcohol consumption was positively associated with performance $(+0.19$ percentage points in total score per $25 \mathrm{~g}$ ethanol increase). The mean IMT, plaque count, non-HDL cholesterol, and femoral PWV were not significantly associated with performance.

In a multivariable model, diabetes was associated with a lower DDT-free recall score $(-2.08$ percentage points, $p<0.001$ ) (Table 2). Alcohol consumption and exercise were associated with higher DDT-free recall scores $(+0.15$ percentage points per $+25 \mathrm{~g}$ ethanol and +1.07 percentage points if exercise at least once/week, $p<0.01$ for each). CVD history and obesity were no longer significantly related to the DDT-free recall score in the multivariable models. However, if diabetes was removed from the multivariable model, CVD history was significant $(-1.64$ percentage points, $p=$ $0.01)$ and obesity was close to significant $(-0.70$ percentage points, $p=0.06$ ). No significant relationship was observed in the EHLS participants between the DDT-free recall total score and high levels of CRP and IL-6 (age- and sexadjusted mean score by the number of high markers: $0=$ $71.6 \%, 1=72.3 \%$, and $2=71.4 \% ; p=0.60$ ). If a history of heavy alcohol use was included in the multivariable model in place of current alcohol consumption, it was not significantly related to the total DDT-free recall score ( $p=0.15$ ).

The overall mean right-left ear difference, or REA, in DDT-free recall scores was 14.7 percentage points (Table 3). Information on the distribution of the right-left ear difference in DDT scores has been previously reported (Fischer et al, 2017). In age- and sex-adjusted models, CVD, diabetes, and a history of heavy alcohol use some time in the past were significantly associated with a greater adjusted mean right-left ear difference (CVD: +2.5 percentage points, $p=0.03$; diabetes: +3.3 percentage points, $p<0.001$; and heavy alcohol use: +1.9 percentage points, $p=0.03)$. Non-HDL cholesterol $(-0.21$ percentage points per $+10 \mathrm{mg} / \mathrm{dL}, p=0.02$ ) and femoral PWV $(+0.36$ percentage points per $1 \mathrm{~m} / \mathrm{sec}, p<0.01$ ) were also significantly related to the right-left ear difference in DDTfree recall scores. Hypertension, obesity, smoking, exercise, mean IMT, plaque, and weekly alcohol consumption were not associated with the difference. In a multivariable model, effect estimates were similar to those in the age- and sexadjusted models (CVD: +3.11 percentage points, $p=0.02$; non-HDL cholesterol: -0.22 percentage points per $10 \mathrm{mg} / \mathrm{dL}, p=0.01$; and femoral PWV: +0.36 percentage points per $1 \mathrm{~m} / \mathrm{sec}, p<0.01$ ) (Table 4 ). However, diabetes and heavy alcohol use were no longer significant. Adjustment for handedness altered the effect estimates very little (CVD: +3.04 percentage points, $p=0.02$; non-HDL cholesterol: -0.22 percentage points per $10 \mathrm{mg} / \mathrm{dL}, p=0.01$; and femoral PWV: +0.37 percentage points per $1 \mathrm{~m} / \mathrm{sec}, p<0.01$ ).

\section{DISCUSSION}

A ssociations between vascular health-related factors and the DDT-free recall total score and right-left ear difference were observed in this cross-sectional study, suggesting that vascular health and central auditory function may be connected. Diabetes was associated with lower DDT-free recall total scores, whereas exercise and alcohol consumption were associated with higher total scores. Effects were modest in size and although statistically significant, they may not be clinically significant. However, in previous cross-sectional work in the same study cohort, a difference of $-1 \%$ in the DDT total free recall score was observed for every +5 years of age (Fischer et al, 2017). Therefore, the 
Dichotic Digits Test/Fischer et al

Table 1. Free Recall DDT Score (\%) Age- and Sex-Adjusted Means by Potential-Related Factors

\begin{tabular}{|c|c|c|c|}
\hline & \multirow[b]{2}{*}{ N (\%) } & \multicolumn{2}{|c|}{ Free Recall (\%) } \\
\hline & & Mean & $p$-Value \\
\hline Overall & $3,655(100.0)$ & 76.7 & - \\
\hline \multicolumn{4}{|l|}{ Categorically measured factors } \\
\hline Cardiovascular disease history & & & $<0.01$ \\
\hline Yes & $343(9.4)$ & 74.9 & \\
\hline No & $3,294(90.6)$ & 76.9 & \\
\hline Hypertension & & & 0.24 \\
\hline Yes & $1,888(51.9)$ & 76.5 & \\
\hline No & $1,752(48.1)$ & 77.0 & \\
\hline Diabetes & & & $<0.0001$ \\
\hline Yes & $512(14.0)$ & 74.1 & \\
\hline No & $3,135(86.0)$ & 77.1 & \\
\hline Obesity (BMI $\geq 30.0 \mathrm{~kg} / \mathrm{m}^{2}$ ) & & & $<0.001$ \\
\hline Yes & $1,785(49.9)$ & 76.1 & \\
\hline No & $1,791(50.1)$ & 77.4 & \\
\hline Smoking status & & & 0.40 \\
\hline Never & $1,882(51.6)$ & 76.7 & \\
\hline Former & $1,351(37.0)$ & 76.8 & \\
\hline Current & $416(11.4)$ & 76.0 & \\
\hline Ever heavy alcohol & & & $<0.001$ \\
\hline Yes & $731(20.5)$ & 75.1 & \\
\hline No & $2,836(79.5)$ & 77.0 & \\
\hline Exercise at least $1 \times /$ week & & & $<0.0001$ \\
\hline Yes & $2,025(55.5)$ & 77.6 & \\
\hline No & $1,622(44.5)$ & 75.5 & \\
\hline Mean IMT (tertiles) & & & 0.24 \\
\hline 1 & $1,201(33.3)$ & 76.3 & \\
\hline 2 & $1,204(33.4)$ & 77.2 & \\
\hline 3 & $1,201(33.3)$ & 76.9 & \\
\hline Plaque count & & & 0.26 \\
\hline 0 & $1,536(43.5)$ & 77.1 & \\
\hline $1-3$ & $1,618(45.8)$ & 76.9 & \\
\hline \multirow[t]{3}{*}{$4-6$} & $377(10.7)$ & 75.9 & \\
\hline & Mean (Standard & \multicolumn{2}{|c|}{ Free Recall (\%) } \\
\hline & Deviation) & $\beta$ & $p$-Value \\
\hline \multicolumn{4}{|l|}{ Continuously measured factors } \\
\hline Alcohol consumption/week ( $\beta$ : per $25 \mathrm{~g}$ ) & $50.8(95.1)$ & 0.19 & $<0.001$ \\
\hline Non-HDL cholesterol ( $\beta$ : per 10 mg/dL) & $143.5(39.1)$ & 0.08 & 0.11 \\
\hline PWV_femoral ( $\beta$ : per $1 \mathrm{~m} / \mathrm{sec})$ & $9.8(2.7)$ & -0.03 & 0.68 \\
\hline
\end{tabular}

diabetes effect found in the present study could be thought of as roughly equivalent to +10 years of age, whereas the exercise effect could be thought of as roughly equivalent to -5 years of age. CVD history and a greater femoral PWV were significantly related to a greater right-left ear difference in free recall scores.

Table 2. Free Recall DDT Score (\%) Multivariable-Adjusted Linear Regression Models

\begin{tabular}{|c|c|c|c|c|c|c|}
\hline & \multicolumn{6}{|c|}{ Free Recall DDT Score (\%) } \\
\hline & \multicolumn{3}{|c|}{ Full $(n=3,538)$} & \multicolumn{3}{|c|}{ Reduced $(\mathrm{n}=3,627)$} \\
\hline & $B(\%)$ & Standard Error & $p$-Value & $\beta$ & Standard Error & $p$-Value \\
\hline Cardiovascular disease history & -1.06 & 0.66 & 0.11 & - & - & - \\
\hline Diabetes & -1.66 & 0.57 & $<0.01$ & -2.08 & 0.54 & $<0.001$ \\
\hline Obesity & -0.52 & 0.38 & 0.17 & - & - & - \\
\hline Exercise at least $1 \times /$ week & 1.05 & 0.38 & $<0.01$ & 1.07 & 0.38 & $<0.01$ \\
\hline Alcohol consumption/week (per 25 g) & 0.15 & 0.05 & $<0.01$ & 0.15 & 0.05 & $<0.01$ \\
\hline
\end{tabular}

Also includes adjustment for age, sex, education, hearing loss severity, and cognitive impairment. 
Table 3. Right Ear Minus Left Ear Free Recall DDT Scores (\%) Age- and Sex-Adjusted Mean Difference by PotentialRelated Factors

\begin{tabular}{|c|c|c|}
\hline & \multicolumn{2}{|c|}{ Free Recall Right-Left Ear Difference (\%) } \\
\hline & Mean & $p$-Value \\
\hline Overall & 14.7 & - \\
\hline \multicolumn{3}{|l|}{ Categorically measured factors } \\
\hline Cardiovascular disease history & & 0.03 \\
\hline Yes & 16.9 & \\
\hline No & 14.4 & \\
\hline Hypertension & & 0.39 \\
\hline Yes & 14.9 & \\
\hline No & 14.3 & \\
\hline Diabetes & & $<0.001$ \\
\hline Yes & 17.5 & \\
\hline No & 14.2 & \\
\hline Obesity $\left(\mathrm{BMI} \geq 30.0 \mathrm{~kg} / \mathrm{m}^{2}\right)$ & & 0.20 \\
\hline Yes & 15.1 & \\
\hline No & 14.2 & \\
\hline Smoking status & & 0.55 \\
\hline Never & 14.4 & \\
\hline Former & 15.1 & \\
\hline Current & 14.3 & \\
\hline Ever heavy alcohol & & 0.03 \\
\hline Yes & 16.2 & \\
\hline No & 14.3 & \\
\hline Exercise at least $1 \times /$ week & & 0.61 \\
\hline Yes & 14.5 & \\
\hline No & 14.9 & \\
\hline Mean IMT (tertiles) & & 0.56 \\
\hline 1 & 15.3 & \\
\hline 2 & 14.5 & \\
\hline 3 & 14.2 & \\
\hline Plaque count & & 0.96 \\
\hline 0 & 14.7 & \\
\hline $1-3$ & 14.7 & \\
\hline \multirow[t]{3}{*}{$4-6$} & 14.3 & \\
\hline & \multicolumn{2}{|c|}{ Free Recall Right-Left Ear Difference (\%) } \\
\hline & $\beta$ & $p$-Value \\
\hline \multicolumn{3}{|l|}{ Continuously measured factors } \\
\hline Alcohol consumption/week ( $\beta$ : per $25 \mathrm{~g}$ ) & -0.04 & 0.65 \\
\hline Non-HDL cholesterol ( $\beta$ : per 10 mg/dL) & -0.21 & 0.02 \\
\hline PWV_femoral ( $\beta$ : per $1 \mathrm{~m} / \mathrm{sec})$ & 0.36 & $<0.01$ \\
\hline
\end{tabular}

These relationships were independent of any confounding effects of age, sex, education, hearing loss severity, and cognitive impairment.

It has been suggested that auditory perception and speech communication are dependent on the interaction between hearing function, central auditory processing, and cognitive function (Humes et al, 2012). Therefore, performance on tests of speech in noise or dichotic listening is likely dependent on all three of these functions. Although vascular health-related factors have been found to be associated with auditory thresholds and cognitive function in previous studies, the current study adds to these findings by focusing on a central auditory processing test. Adjustments for the effects of hearing loss severity and cognitive impairment were made to evaluate the independent relationships of the vascular factors with central auditory processing.

Previous studies reporting a relationship of vascular health-related factors with hearing function, change, and impairment, using audiometric threshold measures, have included prospective (Kiely et al, 2012; Linssen et al, 2014; Nash et al, 2014; Cruickshanks et al, 2015; Fischer et al, 2015) and cross-sectional investigations (Gates et al, 1993; Cruickshanks, Klein, et al, 1998; Bainbridge et al, 2008; Helzner et al, 2011). Among the findings from these studies was a report that high 
Dichotic Digits Test/Fischer et al

Table 4. Right Ear Minus Left Ear Free Recall DDT Scores (\%) Multivariable-Adjusted Linear Regression Models

\begin{tabular}{|c|c|c|c|c|c|c|}
\hline & \multicolumn{6}{|c|}{ Free Recall Right-Left Ear Difference (\%) } \\
\hline & \multicolumn{3}{|c|}{ Full $(n=3,261)$} & \multicolumn{3}{|c|}{ Reduced $(n=3,339)$} \\
\hline & $B(\%)$ & Standard Error & $p$-Value & $\beta$ & Standard Error & $p$-Value \\
\hline Cardiovascular disease history & 3.04 & 1.31 & 0.02 & 3.11 & 1.28 & 0.02 \\
\hline Diabetes & 1.22 & 1.07 & 0.25 & - & - & - \\
\hline Ever heavy alcohol & 0.78 & 0.90 & 0.39 & - & - & - \\
\hline Non-HDL cholesterol (per 10 mg/dL) & -0.23 & 0.09 & 0.01 & -0.22 & 0.09 & 0.01 \\
\hline PWV_femoral (per $1 \mathrm{~m} / \mathrm{sec})$ & 0.35 & 0.14 & 0.01 & 0.36 & 0.14 & $<0.01$ \\
\hline
\end{tabular}

Also includes adjustment for age, sex, education, hearing loss severity, and cognitive impairment.

$\mathrm{GHb}(>12 \%)$ was related to a twofold increased risk of hearing impairment over a 15-year follow-up (Cruickshanks et al, 2015). However, there was not a significant association of hearing impairment risk with diabetes (selfreported doctor diagnosis or a $\mathrm{GHb}>8 \%$ ) or with other levels of GHb; the increased risk was confined to those with high GHb. A cross-sectional study using selfreported doctor-diagnosed diabetes found that individuals with diabetes had an $80 \%$ increased likelihood of having a hearing impairment in the low-mid frequencies and were over twice as likely to have a high-frequency impairment (Bainbridge et al, 2008). Adjustment for cardiovascular, inflammatory, and neuropathic factors attenuated these relationships, although they remained significant (Bainbridge et al, 2010). A third study, a case-control study of type 2 diabetes, found that controls had significantly shorter suprathreshold gap detection thresholds and lower speech thresholds than cases in the Hearing in Noise Test with background noise (Frisina et al, 2006). It is noteworthy that performance on these two tests is dependent not only on auditory thresholds but also on central auditory processing. The current study supports these findings, as it also found higher DDT scores among participants without diabetes.

The present study also found that exercise and alcohol consumption were associated with higher DDT-free recall scores. Research has been conducted investigating possible protective effects of physical activity (Paffenbarger et al, 1978; Folsom et al, 1997; Jakovljevic, 2017) and moderate alcohol consumption (Friedman and Kimball, 1986; Fuchs et al, 1995; Lucas et al, 2005) on vascular health-related conditions and events for many years, but physical activity and alcohol consumption have generally not been widely studied in relation to hearing loss. In previous work related to physical activity, it has been reported that participants who exercised at least once/week had a slightly ( $-1 \mathrm{~dB})$, but significantly, lower $\mathrm{PTA}_{0.5,1,2,4 \mathrm{kHz}}$ at a 5-year followup examination (Fischer et al, 2015). In addition, in a study of self-reported hearing problems, higher physical activity has been found to be protective (Curhan et al, 2013). In two prospective investigations, alcohol consumption was not found to be significantly related to hearing impairment risk (Cruickshanks et al, 2015; Fischer et al, 2015).

The concept of an REA in dichotic listening tests (Kimura, 1961), in which performance is better for material presented to the right ear than the left ear, has been previously studied, but little work has been done investigating factors related to the REA other than age and handedness. The hypothesized explanation for the REA is that through the crossed afferent auditory pathway, the right ear is linked to the left hemisphere of the brain that specializes in verbal processing. Because the left ear signals are routed to the right hemisphere, interhemispheric transfer through the corpus callosum must take place for recognition of verbal stimuli from the left ear. With age and possible age-related neurodegeneration, this transfer may become less effective in a dichotic listening situation (Jerger et al, 1995; Greenwald and Jerger, 2001). A greater REA has been found in older ages (Wilson and Jaffe, 1996; Strouse and Wilson, 1999a,b; Roup et al, 2006; Fischer et al, 2017) perhaps as a result of a more pronounced age effect on left ear performance than right ear performance.

Recently, a greater REA was found to be related to a lower education level and the presence of cognitive impairment (Fischer et al, 2017) which is compatible with early findings, suggesting that as the difficulty of the task increases, the REA increases (Strouse and Wilson, 1999a,b). In the present study, a greater REA was associated with CVD history and higher femoral PWV, a measure of arterial stiffness, findings which are in line with previous work evaluating the relationship between these measures and cognitive functioning. Greater carotid artery IMT, a measure of generalized atherosclerosis, was found to be associated with an increased risk of cognitive impairment over 10 years (Zhong et al, 2012), and a higher Framingham CVD risk score was reported to be related cross-sectionally to lower cognitive test performance in both sexes and prospectively to cognitive decline in men (Kaffashian et al, 2011). A negative association between femoral PWV and cognitive function has been reported whereby a PWV $>12 \mathrm{~m} / \mathrm{sec}$ was associated with lower cognitive test scores (Zhong et al, 2014). Other studies, including prospective investigations, have 
reported a similar negative association with at least some cognitive tests (Waldstein et al, 2008; Mitchell et al, 2011; Watson et al, 2011), although not all have found significant associations (Poels et al, 2007).

The observed relationships in the current study and previous work may be a result of the potential impact of microvascular changes such as small vessel damage and of various pathophysiologic changes accompanying vascular disease including reduced blood flow. For example, internal auditory artery narrowing and demyelination of the eighth cranial nerve along with thickening of the capillaries in the stria vascularis and spiral ganglion atrophy have been found in autopsied diabetic subjects (Bainbridge et al, 2010). The state of hyperglycemia may affect the central nervous system as well as the inner ear (Frisina et al, 2006), and the oxidative damage, greater apoptosis, and disruption of metabolic processes that accompany prolonged hyperglycemia may result in neurodegenerative damage. By contrast, regular physical activity may be beneficial for auditory function through mechanisms such as improved endothelial function and reduced oxidative stress and may provide neuroprotective effects on central auditory processing (Curhan et al, 2013). For example, regular physical activity has been shown to be associated with lower aortic PWV (less stiffening) (Vaitkevicius et al, 1993; Tanaka et al, 1998) perhaps as a result of reduced oxidative stress (Seals et al, 2009). The physiologic consequences of arterial stiffness include reduced blood flow to the brain and small vessel damage (O'Rourke and Safar, 2005).

The strengths of this study include the large size ( $\mathrm{n}=$ 3,655 ) of the study population and the wide age range of the participants (nearly 80-year age span). Information on numerous vascular health conditions and measures was available for evaluation. Similar protocols were followed by trained and certified examiners in the EHLS and the BOSS, so data from the two cohorts could be combined. The test-retest reliability of the DDT has been shown to be good (Strouse and Wilson, 1999a), although the methodology used in the reliability assessment randomly presented one-, two-, and three-digit pair modalities, which is a departure from the methodology used in the present study, and the participants were on average younger (mean age $=49.4$ years), with a larger percentage of men $(72 \%)$ than women in the present study. Although much of the previous work with dichotic listening tests have evaluated ear-specific function, the total of the right and left ear DDT scores was used as one of the outcomes in the current study. The objective of this study was to investigate the relationship between vascular health-related factors and DDT performance on a population level. The total score was a comprehensive measure of overall performance. With respect to limitations, the study was cross-sectional; therefore, temporal relationships cannot be inferred. The significant factors found to be related to the DDT-free recall total score and the right-left ear difference were not the same in the present study, but this lack of consistency may be a reflection of the different aspects of central auditory processing that they each measure. The DDTfree recall total score provides a summary measure of DDT performance. By contrast, as a difference measure, the right-left ear difference does not include information on how well participants performed. For example, one participant could have scored $95 \%$ in the right ear and $90 \%$ in the left ear and another participant could have scored $75 \%$ in the right ear and $70 \%$ in the left ear; both participants had a right-left ear difference of 5\%, but they performed very differently on the test. Therefore, the DDT-free recall total score was evaluated to determine factors associated with overall central auditory processing function. The right-left ear difference was evaluated to determine factors associated with the right ear advantage without regard to the performance level. The difference in findings between the total score and the right-left ear difference with respect to CVD history may also be related to the possibility that CVD and diabetes share a common effect on the DDT-free recall total score; CVD history was significant when diabetes was not included in the multivariable modeling. Finally, although the DDT right eardirected recall was administered, there was not sufficient variability in performance to allow for the investigation of vascular health-related factors.

The present study found a relationship of a number of vascular health-related factors with performance on the DDT-free recall. Relationships were found when evaluating the DDT-free recall total score as well as the right-left ear difference in the DDT-free recall scores. These results suggest that vascular health-related factors, including modifiable behaviors, may play a role in central auditory function, and these factors should be considered as possible predictors of central auditory decline or dysfunction in future prospective investigations.

\section{REFERENCES}

American National Standards Institute (ANSI). (1999) Maximum Permissible Ambient Noise Levels for Audiometric Test Rooms. ANSI S3.1. New York, NY: ANSI.

American National Standards Institute (ANSI). (2010) Specification for Audiometers. ANSI S3.6. New York, NY: ANSI.

American Speech-Language-Hearing Association (ASHA). (1978) Guidelines for manual pure-tone threshold audiometry. ASHA 20: 297-301.

Bainbridge KE, Cheng YJ, Cowie CC. (2010) Potential mediators of diabetes-related hearing impairment in the U.S. population: National Health and Nutrition Examination Survey 1999-2004. Diabetes Care 33:811-816.

Bainbridge KE, Hoffman HJ, Cowie CC. (2008) Diabetes and hearing impairment in the United States: audiometric evidence from the National Health and Nutrition Examination Survey, 1999 to 2004. Ann Intern Med 149:1-10. 
Bond MG, Barnes RW, Riley WA, Wilmoth SK, Chambless LE, Howard G, Owens B. (1991) for the ARIC Study Group. Highresolution B-mode ultrasound scanning methods in the Atherosclerosis Risk in Communities Study (ARIC). J Neuroimaging 1:68-73.

Cruickshanks KJ, Klein R, Klein BE, Wiley TL, Nondahl DM, Tweed TS. (1998) Cigarette smoking and hearing loss: the epidemiology of hearing loss study. JAMA 279:1715-1719.

Cruickshanks KJ, Nondahl DM, Dalton DS, Fischer ME, Klein BE, Klein R, Nieto FJ, Schubert CR, Tweed TS. (2015) Smoking, central adiposity, and poor glycemic control increase risk of hearing impairment. J Am Geriatr Soc 63:918-924.

Cruickshanks KJ, Nondahl DM, Tweed TS, Wiley TL, Klein BE, Klein R, Chappell R, Dalton DS, Nash SD. (2010) Education, occupation, noise exposure history and the 10-yr cumulative incidence of hearing impairment in older adults. Hear Res 264:3-9.

Cruickshanks KJ, Tweed TS, Wiley TL, Klein BE, Klein R, Chappell R, Nondahl DM, Dalton DS. (2003) The 5-year incidence and progression of hearing loss: the epidemiology of hearing loss study. Arch Otolaryngol Head Neck Surg 129:1041-1046.

Cruickshanks KJ, Wiley TL, Tweed TS, Klein BE, Klein R, MaresPerlman JA, Nondahl DM. (1998) Prevalence of hearing loss in older adults in Beaver Dam, Wisconsin: the epidemiology of hearing loss study. Am J Epidemiol 148:879-886.

Curhan SG, Eavey R, Wang M, Stampfer MJ, Curhan GC. (2013) Body mass index, waist circumference, physical activity, and risk of hearing loss in women. Am J Med 126:1142.e1-1142.e8.

Fischer ME, Cruickshanks KJ, Nondahl DM, Klein BEK, Klein R, Pankow JS, Tweed TS, Dalton DS, Paulsen AJ. (2017) Dichotic digits test performance across the ages: results from two large epidemiologic cohort studies. Ear Hear 38:314-320.

Fischer ME, Cruickshanks KJ, Schubert CR, Pinto A, Klein BE, Klein R, Nieto FJ, Pankow JS, Huang GH, Snyder DJ. (2013) Taste intensity in the Beaver Dam Offspring Study. Laryngoscope 123:1399-1404.

Fischer ME, Schubert CR, Nondahl DM, Dalton DS, Huang GH, Keating BJ, Klein BE, Klein R, Tweed TS, Cruickshanks KJ. (2015) Subclinical atherosclerosis and increased risk of hearing impairment. Atherosclerosis 238:344-349.

Folsom AR, Arnett DK, Hutchinson RG, Liao F, Clegg LX, Cooper LS. (1997) Physical activity and incidence of coronary heart disease in middle-aged women and men. Med Sci Sports Exerc 29:901-909.

Folstein MF, Folstein SE, McHugh PR. (1975) "Mini-mental state". A practical method for grading the cognitive state of patients for the clinician. J Psychiatr Res 12:189-198.

Friedman LA, Kimball AW. (1986) Coronary heart disease mortality and alcohol consumption in Framingham. Am J Epidemiol 124: 481-489.

Frisina ST, Mapes F, Kim S, Frisina DR, Frisina RD. (2006) Characterization of hearing loss in aged type II diabetics. Hear Res 211: $103-113$.

Fuchs CS, Stampfer MJ, Colditz GA, Giovannucci EL, Manson JE, Kawachi I, Hunter DJ, Hankinson SE, Hennekens CH, Rosner B. (1995) Alcohol consumption and mortality among women. $N$ Engl J Med 332:1245-1250.

Gates GA, Anderson ML, Feeney MP, McCurry SM, Larson EB. (2008) Central auditory dysfunction in older persons with memory impairment or Alzheimer dementia. Arch Otolaryngol Head Neck Surg 134:771-777.
Gates GA, Anderson ML, McCurry SM, Feeney MP, Larson EB. (2011) Central auditory dysfunction as a harbinger of Alzheimer dementia. Arch Otolaryngol Head Neck Surg 137:390-395.

Gates GA, Beiser A, Rees TS, D'Agostino RB, Wolf PA. (2002) Central auditory dysfunction may precede the onset of clinical dementia in people with probable Alzheimer's disease. J Am Geriatr Soc 50: $482-488$.

Gates GA, Cobb JL, D'Agostino RB, Wolf PA. (1993) The relation of hearing in the elderly to the presence of cardiovascular disease and cardiovascular risk factors. Arch Otolaryngol Head Neck Surg 119:156-161.

Gates GA, Gibbons LE, McCurry SM, Crane PK, Feeney MP, Larson EB. (2010) Executive dysfunction and presbycusis in older persons with and without memory loss and dementia. Cogn Behav Neurol 23:218-223.

Golding M, Taylor A, Cupples L. (2006) Odds of demonstrating auditory processing abnormality in the average older adult: the Blue Mountains Hearing Study. Ear Hear 27:129-138.

Greenwald RR, Jerger J. (2001) Aging affects hemispheric asymmetry on a competing speech task. J Am Acad Audiol 12:167-173.

Hällgren M, Larsby B, Lyxell B, Arlinger S. (2001) Cognitive effects in dichotic speech testing in elderly persons. Ear Hear 22: $120-129$.

Helzner EP, Patel AS, Pratt S, Sutton-Tyrrell K, Cauley JA, Talbott E, Kenyon E, Harris TB, Satterfield S, Ding J, Newman AB. (2011) Hearing sensitivity in older adults: associations with cardiovascular risk factors in the health, aging and body composition study. J Am Geriatr Soc 59:972-979.

Hommet C, Mondon K, Berrut G, Gouyer Y, Isingrini M, Constans T, Belzung C. (2010) Central auditory processing in aging: the dichotic listening paradigm. J Nutr Health Aging 14:751-756.

Hugdahl K. (1995) Dichotic listening: probing temporal lobe functional integrity. In: Davidsson RJ, Hugdahl K, eds. Brain Asymmetry. Cambridge, MA: MIT Press, 123-156.

Humes LE, Dubno JR, Gordon-Salant S, Lister JJ, Cacace AT, Cruickshanks KJ, Gates GA, Wilson RH, Wingfield A. (2012) Central presbycusis: a review and evaluation of the evidence. $J \mathrm{Am}$ Acad Audiol 23:635-666.

Idrizbegovic E, Hederstierna C, Dahlquist M, Rosenhall U. (2013) Short-term longitudinal study of central auditory function in $\mathrm{Alz}$ heimer's disease and mild cognitive impairment. Dement Geriatr Cogn Dis Extra 3:468-471.

Jakovljevic DG. (2017) Physical activity and cardiovascular aging: physiological and molecular insights. Exp Gerontol. Epub ahead of print.

Jerger J, Alford B, Lew H, Rivera V, Chmiel R. (1995) Dichotic listening, event-related potentials, and interhemispheric transfer in the elderly. Ear Hear 16:482-498.

Jerger J, Chmiel R, Allen J, Wilson A. (1994) Effects of age and gender on dichotic sentence identification. Ear Hear 15:274-286.

Kaffashian S, Dugravot A, Nabi H, Batty GD, Brunner E, Kivimäki M, Singh-Manoux A. (2011) Predictive utility of the Framingham general cardiovascular disease risk profile for cognitive function: evidence from the Whitehall II study. Eur Heart $J$ 32:2326-2332.

Kiely KM, Gopinath B, Mitchell P, Luszcz M, Anstey KJ. (2012) Cognitive, health, and sociodemographic predictors of longitudinal 
decline in hearing acuity among older adults. $J$ Gerontol A Biol Sci Med Sci 67:997-1003.

Kimura D. (1961) Cerebral dominance and the perception of verbal stimuli. Can J Psychol 15:166-171.

Klein R, Klein BE, Linton KL, De Mets DL. (1991) The Beaver Dam Eye Study: visual acuity. Ophthalmology 98:1310-1315.

Koohi N, Vickers DA, Lakshmanan R, Chandrashekar H, Werring DJ, Warren JD, Bamiou DE. (2017) Hearing characteristics of stroke patients: prevalence and characteristics of hearing impairment and auditory processing disorders in stroke patients. $J$ Am Acad Audiol 28:491-505.

Linssen AM, van Boxtel MP, Joore MA, Anteunis LJ. (2014) Predictors of hearing acuity: cross-sectional and longitudinal analysis. J Gerontol A Sci Med Sci 69:759-765.

Lucas DL, Brown RA, Wassef M, Giles TD. (2005) Alcohol and the cardiovascular system: research challenges and opportunities. J Am Coll Cardiol 45:1916-1924.

Mitchell GF, van Buchem MA, Sigurdsson S, Gotal JD, Jonsdottir MK, Kjartansson Ó, Garcia M, Aspelund T, Harris TB, Gudnason V, Launer LJ. (2011) Arterial stiffness, pressure and flow pulsatility and brain structure and function: the age, gene/environment susceptibility-Reykjavik study. Brain 134:3398-3407.

Musiek FE, Gollegly KM, Kibbe KS, Verkest-Lenz SB. (1991) Proposed screening test for central auditory disorders: follow-up on the dichotic digits test. Am J Otol 12:109-113.

Nash SD, Cruickshanks KJ, Klein R, Klein BE, Nieto FJ, Chappell R, Schubert CR, Tsai MY. (2013) Long-term variability of inflammatory markers and associated factors in a populationbased cohort. J Am Geriatr Soc 61:1269-1276.

Nash SD, Cruickshanks KJ, Klein R, Klein BE, Nieto FJ, Huang GH, Pankow JS, Tweed TS. (2011) The prevalence of hearing impairment and associated risk factors. Arch Otolaryngol Head Neck Surg 137:432-439.

Nash SD, Cruickshanks KJ, Zhan W, Tsai MY, Klein R, Chappell R, Nieto FJ, Klein BE, Schubert CR, Dalton DS, Tweed TS. (2014) Long-term assessment of systemic inflammation and the cumulative incidence of age-related hearing impairment in the Epidemiology of Hearing Loss Study. J Gerontol A Biol Sci Med Sci 69: 207-214.

O'Rourke MF, Safar ME. (2005) Relationship between aortic stiffening and microvascular disease in brain and kidney: cause and logic of therapy. Hypertension 46:200-204.

Paffenbarger RS Jr, Wing AL, Hyde RT. (1978) Physical activity as an index of heart attack risk in college alumni. Am J Epidemiol 108:161-175.

Poels MM, van Oijen M, Mattace-Raso FU, Hofman A, Koudstaal PJ, Witteman JC, Breteler MM. (2007) Arterial stiffness, cognitive decline, and risk of dementia: the Rotterdam study. Stroke 38:888-892.

Riley WA, Barnes RW, Bond MG, Evans G, Chambless LE, Heiss G for the ARIC Study Group. (1991) High-resolution B-mode ultrasound reading methods in the Atherosclerosis Risk in Communities (ARIC) cohort. J Neuroimaging 1:168-172.
Roup CM, Wiley TL, Wilson RH. (2006) Dichotic word recognition in young and older adults. J Am Acad Audiol 17:230-240.

Schubert CR, Cruickshanks KJ, Fischer ME, Huang GH, Klein R, Tsai MY, Pinto AA. (2015) Carotid intima media thickness, atherosclerosis, and 5-year decline in odor identification: the Beaver Dam Offspring Study. J Gerontol A Biol Sci Med Sci 70: 879-884.

Seals DR, Walker AE, Pierce GL, Lesniewski LA. (2009) Habitual exercise and vascular ageing. J Physiol 587:5541-5549.

Strouse A, Wilson RH. (1999a) Recognition of one-, two-, and threepair dichotic digits under free and directed recall. J Am Acad Audiol 10:557-571.

Strouse A, Wilson RH. (1999b) Stimulus length uncertainty with dichotic digit recognition. J Am Acad Audiol 10:219-229.

Strouse A, Wilson RH, Brush N. (2000) Effect of order bias on the recognition of dichotic digits in young and elderly listeners. Audiology 39:93-101.

Tanaka H, DeSouza CA, Seals DR. (1998) Absence of age-related increase in central arterial stiffness in physically active women. Arterioscler Thromb Vasc Biol 18:127-132.

Vaitkevicius PV, Fleg JL, Engel JH, O'Connor FC, Wright JG, Lakatta LE, Yin FC, Lakatta EG. (1993) Effects of age and aerobic capacity on arterial stiffness in healthy adults. Circulation 88: 1456-1462.

Waldstein SR, Rice SC, Thayer JF, Najjar SS, Scuteri A, Zonderman AB. (2008) Pulse pressure and pulse wave velocity are related to cognitive decline in the Baltimore Longitudinal Study of Aging. Hypertension 51:99-104.

Watson NL, Sutton-Tyrrell K, Rosano C, Boudreau RM, Hardy SE, Simonsick EM, Najjar SS, Launer LJ, Yaffe K, Atkinson HH, Satterfield S, Newman AB. (2011) Arterial stiffness and cognitive decline in well-functioning older adults. J Gerontol A Biol Sci Med Sci 66:1336-1342.

Wichmann MA, Cruickshanks KJ, Carlsson CM, Chappell R, Fischer ME, Klein BE, Klein R, Tsai MY, Schubert CR. (2014) Long-term systemic inflammation and cognitive impairment in a population-based cohort. J Am Geriatr Soc 62:1683-1691.

Wilson RH, Jaffe MS. (1996) Interactions of age, ear, and stimulus complexity on dichotic digit recognition. J Am Acad Audiol 7: 358-364.

Zhan W, Cruickshanks KJ, Klein BE, Klein R, Huang GH, Pankow JS, Gangnon RE, Tweed TS. (2010) Generational differences in the prevalence of hearing impairment in older adults. Am J Epidemiol 171:260-266.

Zhong W, Cruickshanks KJ, Schubert CR, Acher CW, Carlsson CM, Klein BE, Klein R, Chappell RJ. (2012) Carotid atherosclerosis and 10-year changes in cognitive function. Atherosclerosis 224: $506-510$

Zhong W, Cruickshanks KJ, Schubert CR, Carlsson CM, Chappell RJ, Klein BE, Klein R, Acher CW. (2014) Pulse wave velocity and cognitive function in older adults. Alzheimer Dis Assoc Disord 28: $44-49$. 